\title{
Development of a Computer Game "Ecopoly" for Education on Relationship between Environment and Economy
}

\author{
Tasuya Komuro ${ }^{\dagger}$ \\ Department of Industrial and Systems Engineering, Aoyama Gakuin University \\ 5-10-1 Fuchinobe, Chuo-ku, Sagamihara, Kanagawa 252-5258, Japan \\ E-mail: tatsuya-komuro@hotmail.co.jp \\ Toshiyuki Matsumoto \\ Department of Industrial and Systems Engineering, Aoyama Gakuin University \\ 5-10-1 Fuchinobe, Chuo-ku, Sagamihara, Kanagawa 252-5258, Japan \\ E-mail: matsumoto@ise.aoyama.ac.jp
}

Received, June 2, 2011; Revised, July 4, 2011; Accepted, September 1, 2011

\begin{abstract}
It is necessary for humans who have been facing environmental problems to build a sustainable society in which economic activities coexist with nature. To realize such goals, it is essential to promote and enhance environmental education, and to raise global awareness of environmental issues. As a preceding study, a board game "Ecopoly" based on the Kyoto protocol and the estate dealings game, was developed and the validity of environmental education was verified. This study further aims to develop a computer game "Ecopoly", based on the board game which enables us to learn the relationship between environmental problems and economic activities, and to learn more about environmental problems. This computer game enables to concentrate on it by automatic complicated calculations, and to get a higher visual effect compared with the board game. Experimental testing of the game was conducted with 13 college student subjects, and the validity of the game was verified.
\end{abstract}

Keywords: Environmental Problem, Environmental Education, Computer Game, Ecopoly

\section{INTRODUCTION}

In recent years, human beings have been facing various environmental problems such as global warming (Abe, 1998; Murokawa, 2005). In order to implement countermeasures against such problems, the Kyoto Protocol, an international treaty which set out goals for each country to reduce global warming, was adopted at the Third Conference of the Parties (COP3), held in Kyoto in 1997 (Kaneko, 2004; Salisbury, 2005). In the 21st century, humankind is facing an urgent need to build a sustainable society, in which human economic activities coexist with the natural environment. To realize such goals, it is essential to improve and enhance environmental education and help each global citizen to raise awareness of environmental issues. However, it is deemed necessary to develop effective education methods to maintain a high level of interest and motivation among learners upon implementation of environmental education.
Several preceding studies have presented effective educational methods for environmental education in the form of a game (Sugimoto, 2002; Kaneko, 2004; Kawato, 2005; Saito, 2007). For example, Sugimoto et al. proposed a collaborative learning support system using sensing boards in their study. The system enables users to learn about both city planning and environmental issues from a city level standpoint using a board game. Similarly, the game called "My Earth" (My Earth Projects LLC), a game based on trading cards, provides players with the opportunity to learn not only about global environmental linkages between living organisms, global warming and human activity, but also negotiation skills as a collaborative learning experience while having fun. In this way, if it is possible to develop a new game which deals with environmental issues and the surrounding economic activities for environmental education using a form that may look familiar to youths, it may spark interest of young learners in environmental

$\dagger$ : Corresponding Author 
issues.

As a preceding study (Komuro, 2009), the authors have developed a board game "Ecopoly", based on the content of the Kyoto Protocol or the Kyoto Global Warming Pact, and the board game popular throughout the world, "Monopoly" (Suguru, 1999; Axelrod, 2004). The game allows its players to learn about the importance of the balance between environmental and economic concerns, and its educational effects were validated and reported in the study.

Comparing this study with the earlier mentioned other studies, this study may look similar to the one presented by Sugimoto et al., in that it uses the form of a board game to communicate educational messages; the difference lies in that it deals with environment-related economic issues such as forestation, factory construction or emissions trading through a highly popularized form of a game. Similarly, comparing this study and "My Earth", they are both based on popularized games, although this "Ecopoly" uniquely allows players to learn about the importance of balancing environmental issues with economic issues.

To encourage young learners to play the board game "Ecopoly", however, the authors had to consider and overcome some problems, such as complicacy of the calculations of the money a player possesses, $\mathrm{CO}_{2}$ emissions quota and actual emissions-this may keep the players from focusing on playing the game itself-or the time-consuming preparation process of creating the game kits. To solve these problems, the authors reached the idea of computerizing the game.

This study thus aims to create and develop a computer version of the game "Ecopoly" for environmental education purposes, to raise young people's awareness about the relationship between environmental problems and economic activities, and to help them learn about environmental issues. To achieve the goal, first, the authors literally develop a computer game "Ecopoly" based on the board game "Ecopoly." The authors then validate its effect by using the game in a university class, and by conducting a survey among the students who play the game.

\section{BOARD GAME VERSION OF "ECOPOLY"}

\subsection{Objectives and Content of "Ecopoly"}

When too much emphasis is put on environmental protection, economic activities may suffer from stagnation and as a result, modern-day civilized society may have to give up the convenience that has been built up over generations. Whereas too much emphasis on economic activities may cause environmental destruction and the earth may no longer be able to recover the environment by its self-purification effect. To build a sus- tainable society, it is important to understand that there are trade-offs between environment and economy, and to be keen on striking the right balance between them.

The Kyoto Protocol was adopted in 1997 to prevent global warming and to reduce greenhouse gases, including $\mathrm{CO}_{2}$. It is essential that each country works together and cuts emissions to achieve the goal globally. To successfully achieve the goal, it is necessary for global citizens to learn what kinds of economic activities affect or reduce $\mathrm{CO}_{2}$ emissions, what they should do to control the amount of emissions to keep it below the quota, and how to conduct emissions trading by strengthening their negotiation ability.

\subsection{Outline of "Ecopoly"}

The board game "Ecopoly" is a game in which players move their tokens on a map, throwing two dice, using the game kit shown in Figure 1. A turn begins with the rolling of the dice and advancing clockwise starting from the GO corner. The game is played with a time or turn limit. The game also ends when all the players except one go bankrupt and retire from the game. Otherwise, the players keep moving their tokens, and depending on the squares they land on the map, they can buy or sell land or stock. When the players land on special spaces on the map, they have to draw one of the 61 event cards; it provides players with the opportunities to learn about environmental issues. At the beginning of the game, each player is given a certain amount of $\mathrm{CO}_{2}$ emissions quota and emissions. If the player's emissions exceed the allotted emissions quota, the player must pay a penalty when the game terminates. The player can also trade $\mathrm{CO}_{2}$ emissions quota during the game.

To decide the outcome of the game, the penalty is deducted from the gross assets when the game terminates, and the player with the largest net assets becomes the winner. Since there are trade-offs between environment and economy, if a player keeps purchasing land with high property value, it is probable that they have to

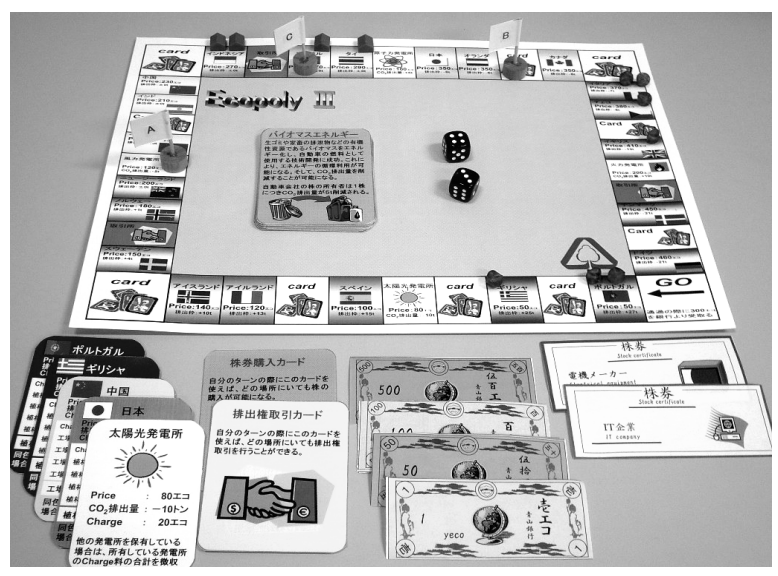

Figure 1. The board game "Ecopoly." 
pay the respective penalty, as their emissions exceed the allotted emissions quotas. In order to win the game, players must consider not only the economic aspects such as purchasing land or increasing their property, but also the environmental aspects such as striking a balance between $\mathrm{CO}_{2}$ emissions quota and emissions.

\section{THE DESIGN OF COMPUER GAME "ECOPOLY"}

By developing the computer version, the game can provide the players with greater visual effects, and it will be easier to deliver the game online that may lead to widespread use of the game.

As for the program development environment, the computer used is DELL's Dimension4590T which utilizes Pentium(R) 4 CPU $2.40 \mathrm{GHz}$, the OS installed is Microsoft Windows XP Professional Edition, and the application development language is Microsoft Visual Basic 6.0.

To develop the computer game, a 3-step procedure is used. First, the game overview flow chart, which shows the overview of the entire system of the game is generated. Next, the game process flow chart, which exhaustively shows how the game proceeds on the board

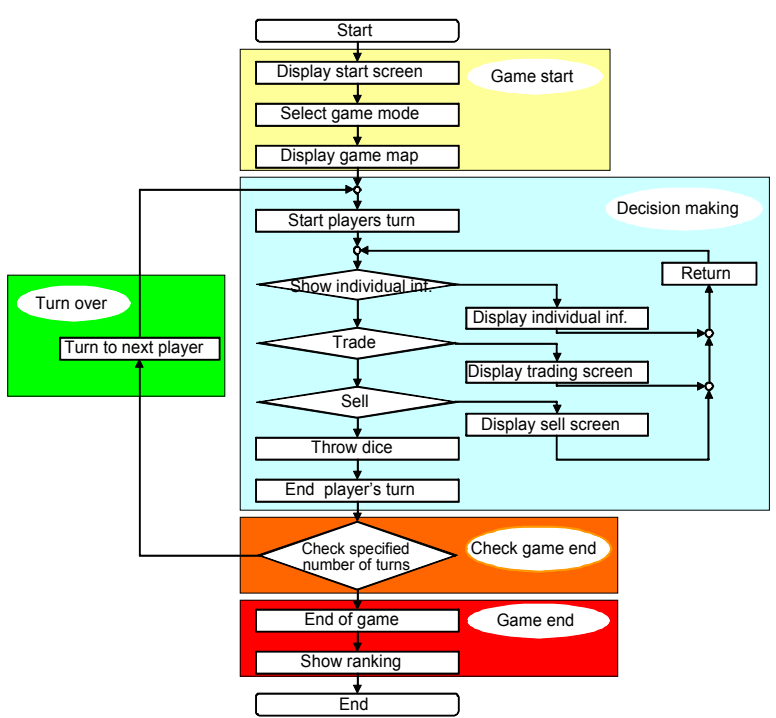

Figure 2. Flow chart.

game is created. Finally, based on the flow charts, the game is programmed.

So, as the first step, the game overview flow cart, the flow chart of the entire system of the game was created. The flow chart is shown in Figure 2, and it shows

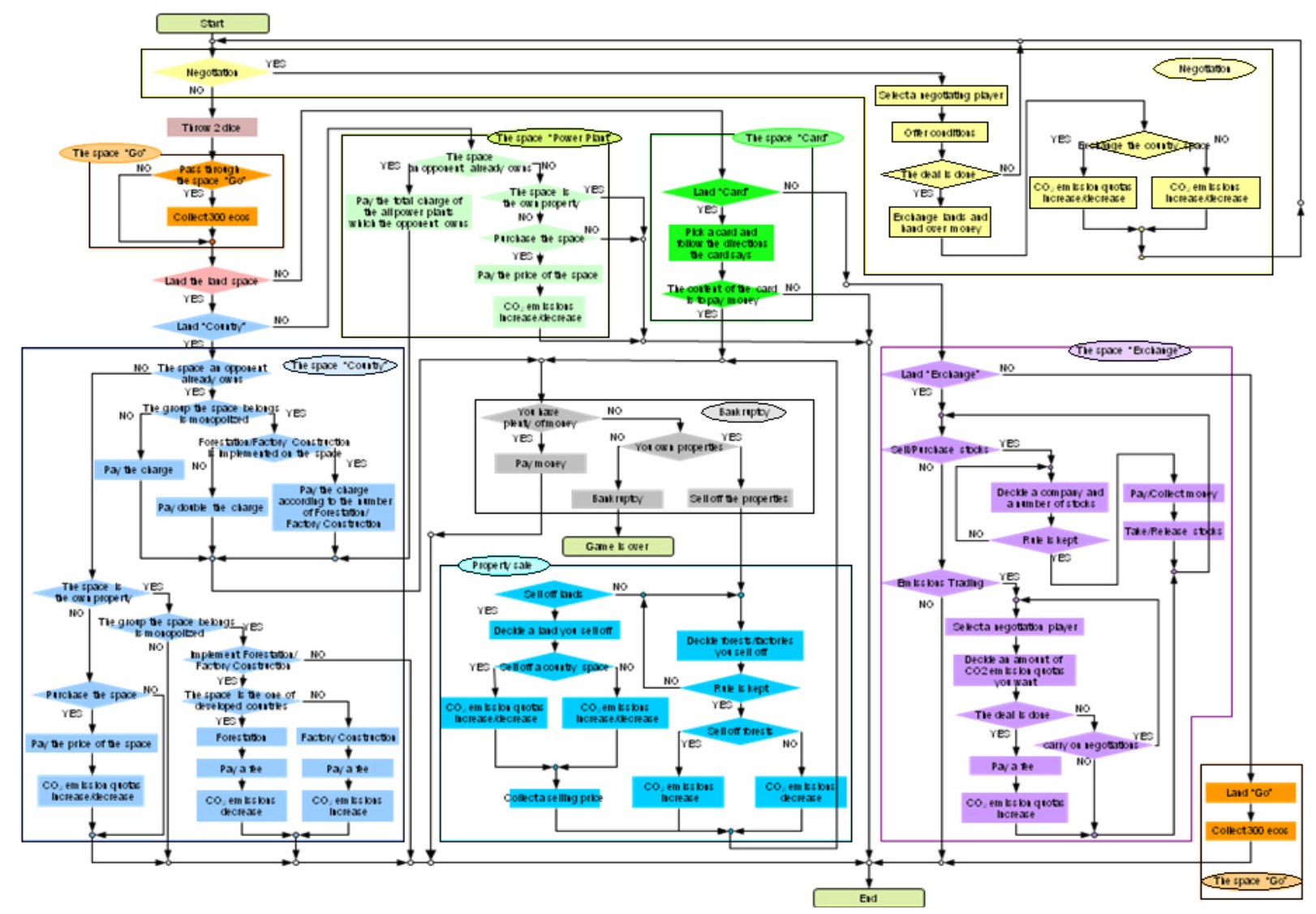

Figure 3. Game Process Flow Chart. 
the entire system to be developed. When playing the board game, players can easily get visual information from the map, for example, the money they own, properties or shares from the items used in the game, such as cards piled in front of the players. If the computer game only focuses on the movement of the tokens and design the system as such, players can not collect various visual information from the screen. Upon systemizing the game flow, the entire system organization was carefully considered and designed taking the points above into consideration.

Next, as the second step, the game process flow chart, which shows how the game proceeds exhaustively was created as shown in Figure 3. This flow chart shows all kinds of decisions that are made when a player's turn comes, and deals are made. This flow chart explains the detailed process of what goes on upon "decision making of a player" described in the game overview flow chart mentioned in step one.

The rules of the computer game are the same as the board game version of the game, and 4 teams compete in a game. The rolling of the dice, movements of the tokens on the board and the cards which provide players with environmental knowledge were all displayed on the screen with the computer version.

In the game, a player needs to deal with properties or $\mathrm{CO}_{2}$ emissions quotas, and negotiate with other players; to promote the deals, personal data of the player and opponents need to be made available. With the computer game, various information is displayed on the screen and made viewable, to make it easier for the players to make various decisions. The money the player possess, $\mathrm{CO}_{2}$ emissions quotas and emissions which had to be manually calculated with the board game version, were all programmed and automatically calculated with the computer version, so that the players can focus more on the tactics of the game while having fun.

\section{THE DEVELOPED COMPUTER GAME "ECOPOLY"}

Computer game "Ecopoly" consists of 21 kinds of input-output display screens and 61 card viewer screens. 9 major screens are shown in Figure 4. The details of each screen are explained below.

When a player starts up the computer game, the opening screen Figure 4(1) is displayed. The game starts by clicking the "start" button.

The main screen shows the map Figure 4(2), and on this map screen, forty squares and spaces are ar-

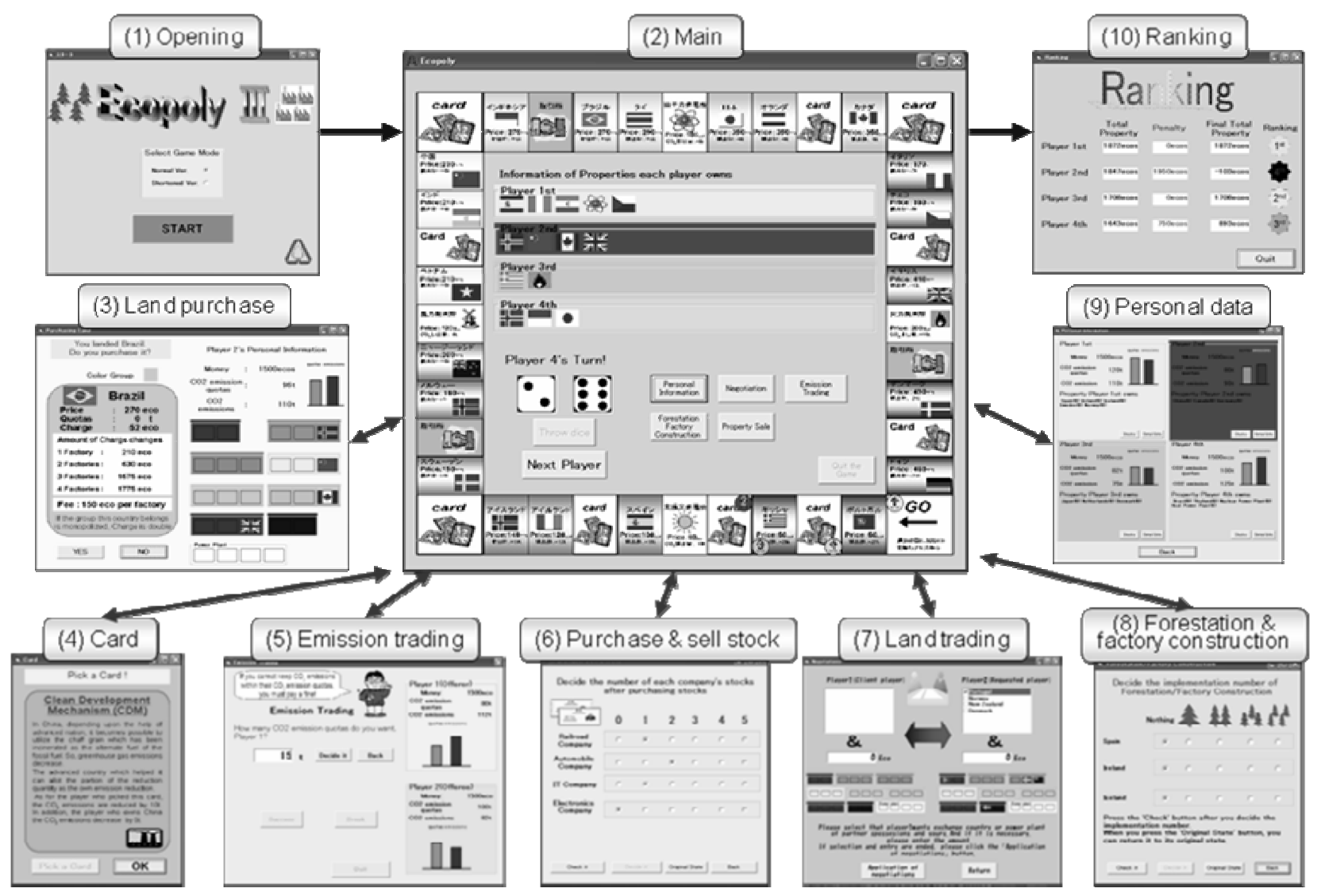

Figure 4. The Main Screen of the Computer Game. 
ranged on the outer border. The 4 colors of the rectangles indicate each player, and the properties the players own are shown with flags in the rectangles. The deal buttons are placed below the rectangles in the mid-lower part of the screen.

The actions players take on this map screen are, rolling the dices, viewing personal data, negotiating with other players, selling properties, forestation, building factories, and quitting the game.

When a player clicks the dice button, the dices roll, and the tokens advance clockwise around the board the corresponding number of the squares the dice show on the map in Figure 5. All the players can select what actions to take and deals to make, except quitting the game. Only the initial player is allowed to select the "quit the game" command, and when the "quit the game" button is pressed, the player has to wait until the opponents finish their turns. To indicate that the player has finished their turn, the player clicks the "next player" button after the "dice" button. The next player's turn begins upon pressing the "next player" button. Functions of other buttons on the map screen will be explained later.

\subsection{When Players Land on Property Squares}

If a player lands on an unowned property, the land purchase screen Figure 4(3) appears. This screen shows the color-group, property land cards, the money the player possesses, the $\mathrm{CO}_{2}$ emissions quota, the emissions, and the status of the property squares the players own. Based on the information shown on this screen, the player decides if they want to purchase the property or not. When a player decides to purchase the property, they press the "Yes" button. If the player declines the purchase, they press the "No" button. When either but-

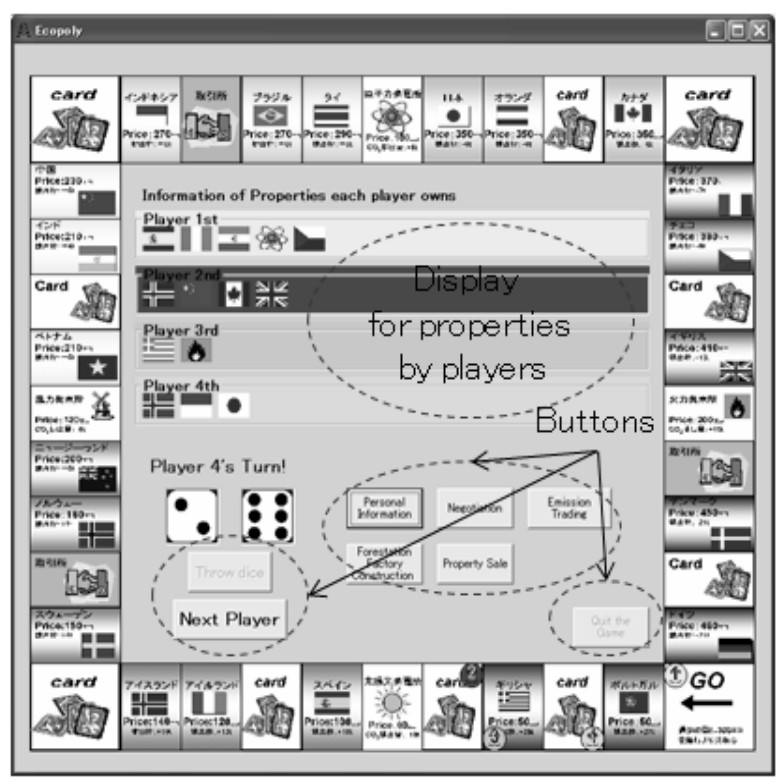

Figure 5. Buttons in the Map. ton is pressed, the game continues and they go back to the main map screen. When the player purchases properties, respective flags of the land they have purchased are added to the colored rectangles which show the "status of property squares."

If the property landed on is already owned by other players, the player must pay charge, so the "charge collection" screen appears. This screen shows the property squares, the forests, and factories the player own. Players must press the "OK" button to exit. If the player possesses enough cash on hand, the charge is deducted and the player goes back to the map screen. If the player does not possess adequate funds in cash, a bankruptcy warning screen appears. When the player owns properties they can sell off, they may press the "sell" button to show the "sell screen", and sell off their properties. After selling, the bankruptcy warning screen appears again; the player presses the "pay the debt" button, pay the charge and go back to the map screen. If the player possesses no properties they can sell off, the player must press the "bankruptcy" button and retire from the game.

\subsection{When Players Land on Card Squares}

When a player lands on card squares, the card screen Figure 4(4) appears. By pressing the "draw a card" button on the card screen, the selected card is displayed in the center of the screen. The 61 cards provide players with the opportunity to learn about environmental issues. The player reads the card, and then presses the "OK" button to exit and follows the instructions on the cards, as they do when playing the board game version of the game. Here, the game does not provide its intended educational effect if the players do not read the cards carefully. The authors would like to consider adding some functions or a comprehension check device, such as a voice service, as a future reissue.

\section{3 When Players Land on Exchange Squares}

When players land on an exchange land, the exchange screen appears. On the exchange screen, players can conduct trade of 2 kinds of items, $\mathrm{CO}_{2}$ emissions and stock.

When a player wants to trade emission rights, they press the "negotiate" button. Upon pressing the button, an emissions trading screen Figure 4(5) appears. On this screen, the player that wants to make a deal presses the "negotiate" button. Upon pressing the button, an emissions trading screen appears, and the player enters the amount of $\mathrm{CO}_{2}$ emission quota they wish to purchase. The player then presses the "confirm" button to start the deal, and if they change their mind at this point, they may press the "return" button to go back to the map screen. After the "confirm" button is clicked and the negotiation starts, the opponent who is asked to make a deal on the emission right presses either the "accept" or "decline" button. If the opponent accepts the deal, the 
money the players possess and the amounts of $\mathrm{CO}_{2}$ emissions quotas shown on the right side change accordingly. When the deal finishes, the player presses the "return" button and goes back to the exchange screen.

To buy or sell stock, players press a "purchase stock" or "sell stock" button on the exchange screen. When the buttons are pressed, the stock trading screen Figure 4(6) appears. Players select which stock they wish to purchase or sell, and how many shares they would like to purchase or sell, by entering the numbers of shares of each company. After entering the number, players press the "check" button to confirm or correct the details of the deals the players wish to make, and the players either continue the deals by pressing the "confirm" button or make necessary corrections if any. When the "reset" button is pressed, the deal is canceled and the initial status is retrieved. After the deals, players go back to the map screen by pressing the "return" button.

\subsection{Land Trading}

When a player presses the "negotiate" button on the map screen, the opponent selection screen appears, and the player can select the opponent they want to negotiate with. After the player selects the opponent, they press the "confirm" button to show the land trading screen Figure 4(7). Then the player either selects the property land they wish to exchange, or enters the amount of money they offer to pay for the land using the keyboard, if they prefer monetary exchange. After selecting and completing the form on the screen, the player presses the "start negotiation" button. The opponent who is asked to negotiate presses the "accept" or "decline" button, and shows if they are willing to close the deal. If the opponent accepts the deal, the money the players possess and the amounts of $\mathrm{CO}_{2}$ emissions quotas change accordingly. When the deal finishes, the player presses the "return" button, and goes back to the map screen.

\subsection{Forestation or Factory Construction}

22 property spaces on the map which represent countries consist of 8 color-groups. Once a player owns all properties of a color-group and monopolizes the color-group, the charge is doubled and the player may either plant trees or build factories.

To plant trees or build factories, a player presses the "forestation" or "build factories" button. Upon pressing the buttons, a property selection screen for forestation or factory construction appears. The player then selects the color-group they wish to plant trees or build factories on. When the color-group is not monopolized, the player cannot press these buttons, and they must return to the map screen by pressing the "return" button.

When a color-group for forestation or factory construction is selected, the forestation or factory construction screen Figure 4(8) appears. The player chooses the total number of forests or factories after the deal, and presses the "check" button. After confirming the details, the player presses "confirm" to complete the deal, and to correct, select the information again. To retrieve the initial status, press the "reset" button. After forestation or factory construction, the player presses the "return" button to go back to the map screen.

\subsection{Selling Properties}

When a player presses the "sell properties" button on the map screen, a property selection screen appears and the player can select the properties they wish to sell. If they change their minds, they can press the "return" button and go back to the map screen.

If the property the player wants to sell is a property land which represents a country, or a power station, they press the "country or power station" button on the property selection screen. If the player possesses property lands, the list of the squares they own is displayed, and the player selects the property land they wish to sell. Here, it should be noted that the property land with forests or factories are not added to the list. After the player selects the property, they press the "check" button, and select whether or not they would like to sell the selected land. If they wish to sell the property, they can press the "confirm" button. To change the details, they select the properties again. After selling, they press the "return" button to go back to the map screen.

If the property the player wants to sell is a forest or factory, they press a "forest or factory" button on the property selection screen. When the button is pressed, the selection screen for the properties with forests or factories appears, and the player selects the color-group of the forest or factory they wish to sell. When the player does not possess color-groups they monopolize, they cannot select any color-groups. If the player changes their mind at this stage and does not wish to sell the properties, they press the "return" button and go back to the map screen.

When a color-group is selected, a forest or factory selection screen appears, and the player conducts the transaction by entering the number of forests or factories they are to possess after the transaction, then presses the "check" button. To finalize the deal, the player presses the "confirm" button; otherwise the player re-enters the total number of forests or factories they are to possess after the deal again. To go back to the initial status, and undo the changes in the number of forests or factories, they press the "reset" button. After the transaction, or to cancel the deal, they press the "return" button and go back to the map screen.

\subsection{Personal Data}

When a player presses the "personal data" button on the map screen, the personal data screen Figure 4(9) appears. The screen shows the details of each player's personal financial data, such as the money the player has 
in assets, the $\mathrm{CO}_{2}$ emissions quota and emissions, and the properties the player own. The screen is linked to the detail screen and the stock screen, and the respective data is shown when the buttons are pressed. To go back to the personal data screen and view personal data, players press the "close" and "return" buttons. Using these functions, players can visually compare the data shown on the screen, which makes it easier for players to make various decisions.

\subsection{Landing on or Passing GO Square}

When dice are rolled and a player lands on or passes GO square, the salary payment screen appears. When a player has confirmed the message, they press the "OK" button to go back to the map screen. Upon pressing the "OK" button, 300 ecos are added to the player's account.

\subsection{End Game}

The game ends when the specified time is up, or when players take the specified number of turns. The game can also come to an end when everyone except one player goes bankrupt. When the game finishes, the player presses the "end game" button, and when the button is pressed, an "end" screen appears. The player then presses a "ranking" button to show the players' rankings Figure $4(10)$. When the button is pressed, a ranking screen appears, and the players are ranked according to the net worth of each player. The players' gross assets, penalties, and the net assets are presented, and the ranking is announced. The game terminates when the "finish" button is pressed after the announcement of the players' ranking.

\section{EVALUATION OF THE COMPUTER GAME}

To verify the educational effect of the developed computer game "Ecopoly" with the board game version, an experiment was conducted in a lesson at college, having 13 students, both male and female, as subjects as shown in Figure 6.

Before the experiment, each subject was given a game rule book, and was asked to read it in advance. In the lesson, first, a quiz was given (5 min). Next, there was a lecture on the global environmental issues and an outline of the Kyoto Protocol, followed by an explanation of the outline of the game "Ecopoly" and its rules (30 min). After the explanation, the subjects were asked to play the board game version of Ecopoly $(55 \mathrm{~min})$, reported in the preceding study, and then the subjects played the computer game version of Ecopoly (15 min), presented in this study. After the sessions, there was an explanation on the relationship between the environment

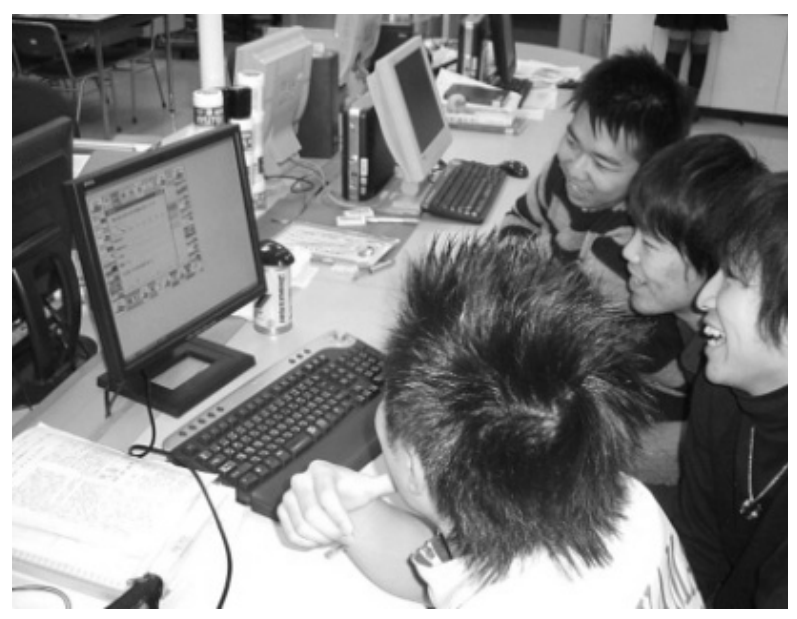

Figure 6. Implementation of the Game.

and the economy, as it is the central concept of the game design, and finally the subjects were asked to answer a quiz and a questionnaire survey (20 min).

The quiz consists of 10 multiple choice questions, and the subjects were asked to work on the quiz before and after the experimental session, to verify the educational effects of Ecopoly. During the game, the subjects have opportunities to draw cards, and the content of these cards are related to the content of the quiz. If the subjects read the cards carefully and understand the content, they can select the correct answers for the quiz. The result of the experiment shows that the average percentage of those questioned answered correctly among the 13 subjects rose from 5.5 questions before the experiment to 7.0 questions after the experiment. Also, the result of the t-test stands at a significant level of $1 \%$, and shows a significantly higher level $\left(\mathrm{t}(12)=3.7^{* *}\right)$, and the educational effects of the game is verified. However, no subject answered all the questions correctly, so it still has significant scope to continue to improve for the future. In the preceding study with only the board game version the same quiz was conducted for 25 high school students. The average percentage of those questioned answered correctly rose from 5.5 to 7.1 questions after the experiment. The result of these two experiments of the $t$-test shows no significance $(t(36)=0.4)$.

In the questionnaire survey, awareness of environmental issues, the lecture and the game were evaluated by the subjects. The questions are identical with the ones used and reported in the preceding study. All the subjects answered "I could understand the relationship between environment and economy better", and 93\% (12 subjects) answered "I became more interested in the environmental issues in general." As for the evaluation of the computer game itself, all answered "I could enjoy playing the game."

In conclusion, although the game session computer version of "Ecopoly" was shorter than that of the board game version by 40 minutes, and tested with different generation of subjects, as the board game subjects were 
high school students and the subjects of this study were college students, it is still possible to conclude that the computer game version can provide equally effective educational effects compared with the board game version.

\section{CONCLUSIONS}

In this study, the computer game version of "Ecopoly" based on its board game version presented in the authors' earlier study, was developed and presented. "Ecopoly" enables the users to learn about both environmental issues and economic activities, and based on the popular board game "Monopoly", it deals with the content of the Kyoto Protocol. The developed computer game was used in an experiment session, joined by 13 college students as subjects, and the educational effects of the game was verified. Compared with the board game version, the computer version of the game enables the users to concentrate on the content and strategies of the game, because the players do not have to deal with the complicated calculation of the money they possess, $\mathrm{CO}_{2}$ emissions quotas, emissions, and changes in these values. The computer game can also provide the users with higher visual effects, and by computerizing the game, it was made possible to distribute the game online and to popularize the game as a new and effective educational tool on the environment and economy.

The authors would like to face the challenges of solving the ways to distribute the developed program online, and to design and program algorithms for NPC (Non-Player Character), to enable individual learning using this program in the future. Also the authors have a plan to develop other education materials which treat ecological behaviors, techniques of $\mathrm{CO}_{2}$ calculation, or the real world scenario on environmental issues.

\section{ACKNOWLEDGEMEZNT}

This study is funded by Grant-in-Aid for Scientific Researches (numbered 20500785). The authors would like to express their gratitude to the organization.

\section{REFERENCES}

Abe, K. et al. (1998), An Outline of Global Environment (in Japanese), University of Tokyo Press, ISBN 413-062139-4.

Axelrod, A. (2004), Everything I Know About Business I Learned From Monopoly (in Japanese), DIAMOND-SHA, ISBN 0-7624-1694-7.

Kaneko, K., Fujita, K., and Baba, M. (2004), Recommendation of New Environmental Education (in Japanese), Nikkei Ecology, 10, 24-37.

Kawato, M. and Chikamori, K. (2005), A Study of Environmental Education for Energy, Journal of Conference on Energy, Economy, and Environment, 21, 239-242.

Komuro, T. and Matsumoto, T. (2009), Development of a Board Game "Ecopoly" for Education on Relationship between Environment and Economy (in Japanese), Transactions of Japanese Society for International and Systems in Education, 26(4), 339348.

Meadows, D. H. et al. (1972), The Limits to Growth, Chelsea Green Publishing, ISBN 978193149858.

Murokawa, I. (2005), Trend and Measures of Kyoto protocol; What is going on Post Kyoto (in Japanese), Nikkei Ecology, 4, 156-160.

My Earth Projects LLC, My Earth, http://myearth.ne.jp.

Saito, S. and Yamane, K. (2007), Evolving Environmental Education (in Japanese), Nikkei Ecology, 6, 2437.

Salisbury, L., Zucker, P., Tazaki, H., and Yamazoe, M. (2005), Delicate Relationship between $\mathrm{CO}_{2}$ Emissions Quota and Post Kyoto (in Japanese), Nikkei Ecology, 7, 128-129.

Sugimoto, M., Kusunoki, F., Inagaki, S., Takatoki, K., and Yoshikawa, A. (2002), A System for Supporting Collaborative Learning with Networked Sensing Boards (in Japanese), Journal of the Institute of Electronics, Information and Communication Engineers, J85-D1(12), 1152-1163.

Suguru, K. (1999), MONOPOLY Club (in Japanese), TBS-BRITANNICA, ISBN 4-484-99216-7. 